\title{
Troponin T predicts in-hospital and 1-year mortality in patients with pulmonary embolism
}

\author{
K.M. Janata*, J.M. Leitner*, N. Holzer-Richling*, A. Janata*, \\ A.N. Laggner* and B. Jilma
}

ABSTRACT: We aimed to determine the prognostic value of troponin T (TNT) for in-hospital and 1yr mortality in a large sample of patients with pulmonary embolism (PE).

Patients presenting at the emergency department of a tertiary care centre from January 1998 to December 2006 with PE were included. A blood sample was taken at the time of presentation. To determine in-hospital and 1-yr mortality, data from the hospital records and the national death register were used.

TNT was determined in 563 out of 737 patients with proven PE. TNT was elevated $\left(>0.03 \mathrm{ng} \cdot \mathrm{mL}^{-1}\right)$ in $27 \%$. In-hospital survival was $79 \%$ in TNT-positive patients compared with $94 \%$ in TNT-negative patients $(p<0.001)$. 1-yr survival was $71 \%$ in TNT-positive patients compared with $90 \%$ in TNT-negative patients $(p<0.001)$. Elevated TNT levels meant a four-times higher risk of in-hospital death and a three-times higher risk of 1-yr mortality, even after adjustment for the other most important risk factors of death in this population.

Elevated TNT independently predicts in-hospital and 1-yr mortality in patients with acute PE.

KEYWORDS: Prognosis, pulmonary embolism, troponin

C ardiac troponins are highly specific and sensitive markers of myocardial cell injury and have been found to be elevated in acute coronary syndromes, myocarditis, acute heart failure, septic shock and after therapeutic cardiac procedures [1]. Pulmonary embolism (PE) is associated with elevated cardiac troponins in only a subset of patients. The reason for release in PE is still unclear. However, an explanation might be hypoxaemia due to perfusionventilation mismatch, hypoperfusion as a consequence of low output and reduced coronary blood flow, as well as cell injury caused by acute dilatation of the right ventricle, or a combination of these factors.

Currently, prognostic stratification in PE is based on blood pressure at admission, troponins as markers of right ventricular ischaemia and right ventricular dysfunction mainly assessed by echocardiography $[2,3]$. However, the lack of standard criteria for right ventricular dysfunction and the need for specialised personnel limit the use of echocardiography. Troponins, which are rapidly available, have been shown to be associated with right ventricular dysfunction in PE. While their use seems to be marginal in diagnosis, troponins may significantly contribute to prognostic stratification [4]. Due to their high negative predictive value for mortality in PE, they are particularly useful for the identification of lowrisk patients [5]. Investigations are ongoing to evaluate whether troponins might qualify as a tool for the identification of a certain portion of haemodynamically stable patients who would benefit from thrombolysis. Currently, thrombolysis is only recommended in patients with massive PE causing haemodynamic instability [6].

In a recent meta-analysis [7], elevated plasma levels of troponins were associated with increased in-hospital or 3-month mortality in patients with acute PE. In the 20 included studies (eight evaluating troponin $\mathrm{T}$; TNT), the longest observation period was 100 days and the average study comprised of 98 patients. However, data regarding clinical outcome is scarce and the true predictive value of elevated troponins for adverse outcome is still unclear. Thus, our study aimed to determine the relationship between TNT and survival in a much larger sample of patients diagnosed with PE. Compared to the studies included in the meta-analysis, our study clearly comprises the largest number of patients and the longest observation period.
AFFILIATIONS

Depts of *Emergency Medicine,

\#Infectious Diseases and Tropical Medicine, and

"Clinical Pharmacology, Medical University of Vienna, Vienna, Austria.

CORRESPONDENCE

K.M. Janata

Dept of Emergency Medicine Medical University of Vienna Waehringer Guertel 18-20 1090 Vienna

Austria

E-mail:

karin.janata@meduniwien.ac.at

Received:

Feb 252009

Accepted after revision:

June 042009

First published online:

June 182009 
TABLE 1 Patient characteristics

Total of patients with PE Patients without TNT TNT-positive patients TNT-negative patients $\quad p$-value ${ }^{\#}$ measurement

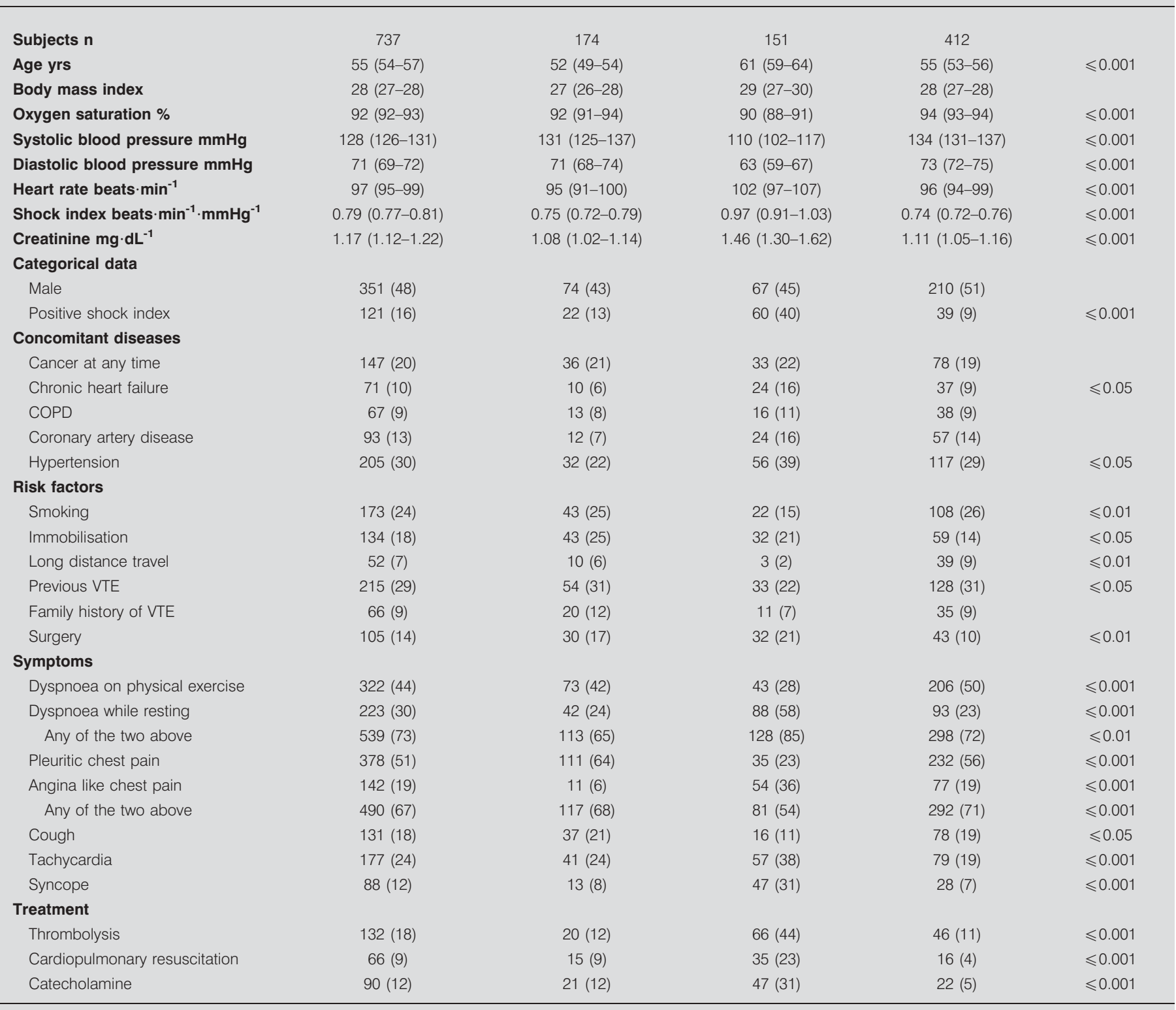

Data are presented as mean (95\% confidence interval) or $\mathrm{n}(\%)$, unless otherwise stated. PE: pulmonary embolism; TNT: troponin T; COPD: chronic obstructive pulmonary disease; VTE: venous thromboembolism. ${ }^{*}$ : p-values are provided for descriptive purposes only and are between TNT positive and TNT negative patients.

\section{METHODS}

\section{Patients and measurements}

All patients diagnosed with PE from January 1998 to December 2006, who had at least one blood collection for TNT measurement at the time of presentation at the emergency ward of Vienna General Hospital (Vienna, Austria), a tertiary care facility, were included. Diagnosis of PE had to be definite, either by ventilation-perfusion scintigraphy, spiral computed tomography, angiography, thrombus detection by trans-oesophageal echocardiography or autopsy. A number of patients $(n=106)$ were included in a previously published study evaluating in-hospital mortality [8]. The measurement of TNT was routinely performed by electrochemiluminescence immunoassay on an Elecsys 2010 analyser (Roche Diagnostics AG, Rotkreuz, Switzerland) (coefficient of variation: 5.7\%) after blood sampling into evacuated tubes for clinical chemistry analysis (Vacutainer tubes; Becton Dickinson, Heidelberg, Germany). A TNT value of $>0.03 \mathrm{ng} \cdot \mathrm{mL}^{-1}$ was interpreted as "positive". All data except outcome were continuously entered into the database. To determine in-hospital and 1-yr mortality, a double data set of the hospital records and the Austrian death register were used. Deaths were classified by International 

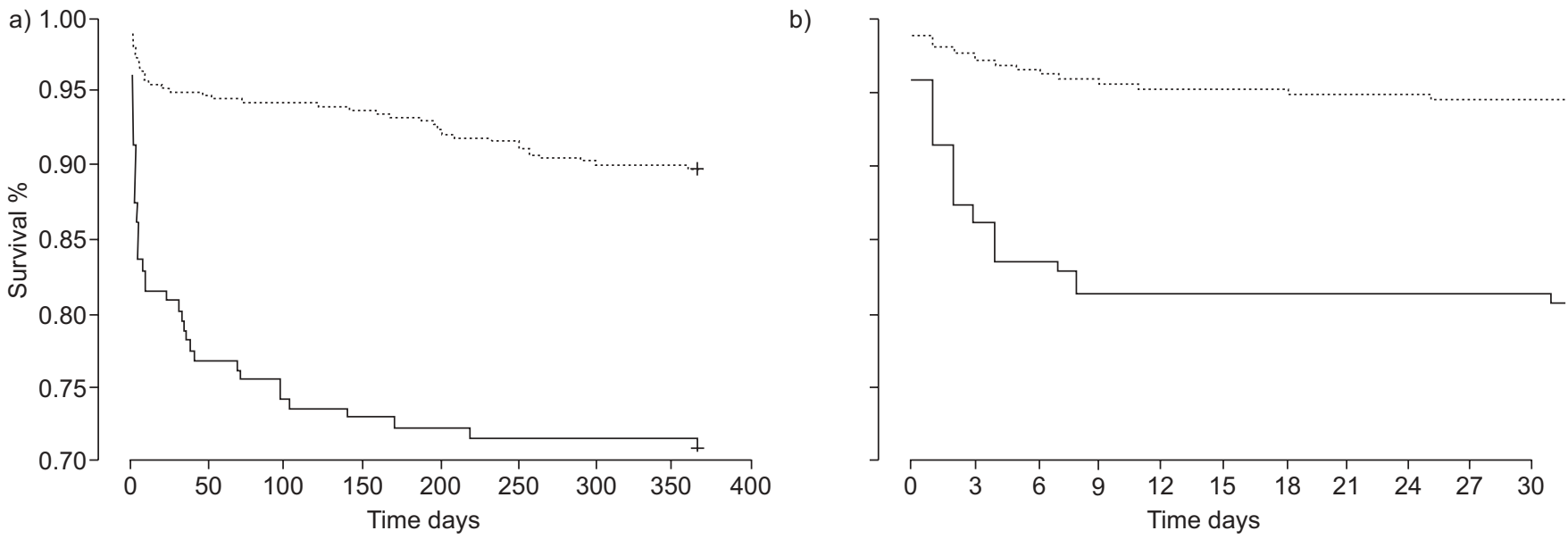

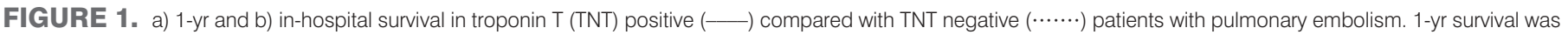

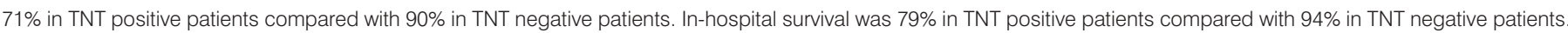

Classification of Disease codes in both cases. In seven inconsistent classification cases a decision was made by a single investigator from the case history without regard of the TNT value. Pathologists and clinicians classifying causes of death did not know about the inclusion of the patients into the study.

\section{Statistical analysis}

Mean and 95\% confidence intervals are provided for descriptive analysis and non-parametric tests were used for reasons of robustness. Survival rates are plotted according to the method of Kaplan-Meier, and log rank and Breslow tests were calculated. Multivariate analysis was performed with the Cox regression, which also included cancer, immobility and age as known risk factors $[9,10]$ for short-term mortality in patients suffering from PE. The level of significance was set to a twosided probability value $(\mathrm{p}<0.05)$. Data were analysed using SPSS Version 14.0 (SPSS Inc., Chicago, IL, USA). For generation of the Forrest plot, Review Manager 5.0.16 (Cochrane Collaboration, Oxford, UK) was used.

\section{RESULTS}

TNT was determined in 563 out of 737 patients diagnosed with PE. In $151(27 \%)$ patients TNT levels were $>0.03 \mathrm{ng} \cdot \mathrm{mL}^{-1}$, while $412(73 \%)$ patients were TNT negative. TNT-positive patients were older, had more comorbidities, higher serum creatinine levels and were more likely to present with severe clinical signs and symptoms of $\mathrm{PE}$, including angina-like chest pain, dyspnoea while resting, tachycardia, low oxygen saturation and syncope. During the initial phase they were more likely to need cardiopulmonary resuscitation, catecholamines or to undergo thrombolysis (table 1).

During 1 yr of follow-up, 112 (15\%) out of the 737 patients died. The mortality rate was similar in the $174(15 \%)$ patients who had no TNT determined. 44 (29\%) of the TNT-positive

TABLE 2 Mortality and causes of death in the study population

Total of patients with PE

Patients without TNT measurement

\begin{tabular}{|c|c|c|c|c|}
\hline Subjects $\mathbf{n}$ & 737 & 174 & 151 & 412 \\
\hline \multicolumn{5}{|l|}{ Outcome } \\
\hline Death in the first year & $112(15.2)$ & $26(14.9)$ & $44(29.1)$ & $42(10.2)$ \\
\hline In hospital death & $73(9.9)$ & $17(9.8)$ & $32(21.2)$ & $24(5.8)$ \\
\hline \multicolumn{5}{|l|}{ Causes of death } \\
\hline Pulmonary embolism & $48(6.5) ; 43$ & $15(8.6) ; 58$ & 23 (15.2); 52 & $10(2.4) ; 24$ \\
\hline Cancer & $28(3.8) ; 25$ & $3(1.7) ; 12$ & $8(5.3) ; 18$ & $17(4.1) ; 40$ \\
\hline Coronary artery disease & $3(0.4) ; 3$ & $0(0.0) ; 0$ & $1(0.7) ; 2$ & $2(0.5) ; 5$ \\
\hline Heart failure & $4(0.5) ; 4$ & $1(0.6) ; 4$ & $2(1.3) ; 5$ & $1(0.2) ; 2$ \\
\hline Intracerebral haemorrhage & $1(0.1) ; 1$ & $0(0.0) ; 0$ & $0(0.0) ; 0$ & $1(0.2) ; 2$ \\
\hline Stroke & $1(0.1) ; 1$ & $1(0.6) ; 4$ & $0(0.0) ; 0$ & $0(0.0) ; 0$ \\
\hline Other & $9(1.2) ; 8$ & $2(1.1) ; 8$ & $5(3.3) ; 11$ & $2(0.5) ; 5$ \\
\hline Unknown & $18(2.4) ; 16$ & $4(2.3) ; 15$ & $5(3.3) ; 11$ & $9(2.2) ; 21$ \\
\hline
\end{tabular}

\section{Subjects $\mathbf{n}$}

hospital death

Pulmonary embolism

Cancer

Heart failure

Intracerebral haemorrhage 

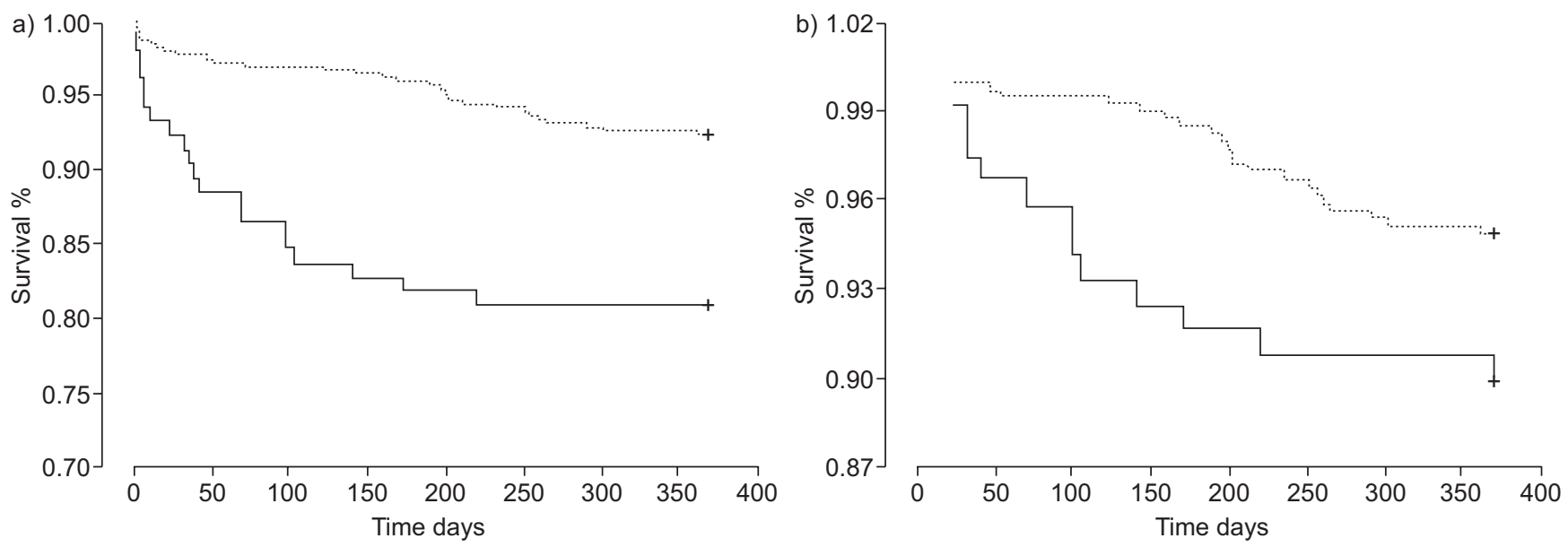

FIGURE 2. 1-yr survival after a) exclusion of initially haemodynamically instable patients and b) exclusion of patients who died in hospital. Patients who were resuscitated prior to or at admission, who presented with a systolic blood pressure $<90 \mathrm{mmHg}$, or who were treated with catecholamines in the pre-clinical phase were excluded. In the initially haemodynamically stable patients, 1 -yr survival was $81 \%$ in troponin T (TNT) positive patients compared with $92 \%$ in TNT negative patients. After exclusion of patients who died in hospital, 1-yr survival was 90\% in TNT positive patients compared with 95\% in TNT negative patients. ——: TNT positive patients; $\cdots \cdots . . . \cdot$ TNT negative patients.

patients died compared to $42(10 \%)$ of the TNT-negative patients (Rank and Breslow test: $\mathrm{p}<0.001$ ) (fig. 1a). The inhospital death rate was $10 \%$ in the overall study population and patients without TNT measurement, 32 (21\%) of the TNTpositive patients and $24(6 \%)$ of the TNT-negative patients (Rank and Breslow test: $\mathrm{p}<0.001$ ) (table 2, fig. 1b). Exclusion of patients who were unstable at presentation resulted in 1-yr mortality rates of $19 \%$ and $8 \%$ in TNT-positive and TNTnegative patients, respectively $(p<0.001$; fig. $2 a)$. After exclusion of patients who died in hospital, the median duration of hospitalisation was 11 days. The median duration of hospitalisation was 14 days in TNT-positive patients compared with 10 days in TNT-negative patients. Patients who survived the hospitalisation phase had a 1 -yr mortality rate of $10 \%$ and $5 \%$, TNT-positive versus TNT-negative patients, respectively ( $\mathrm{p}=0.047$; fig. $2 \mathrm{~b})$.

The major cause of death in the first year was PE in TNTpositive patients and in patients without TNT measurement, while in TNT-negative patients the cause of death was cancer (table 2). Of the 48 patients who died from PE, 45 died in hospital. In contrast, of the 28 patients who died from cancer, only 11 died in hospital. Other reasons for death such as congestive heart failure, coronary artery disease, stroke and intracerebral haemorrhage were less common. In $18(16 \%)$ patients the reason for death was not specified in the death register and was, therefore, classified as unknown.

Elevated TNT levels $\left(>0.03 \mathrm{ng} \cdot \mathrm{mL}^{-1}\right)$ were associated with a significant increase in the risk of all-cause death in hospital (HR 4.3, 95\% CI 2.5-7.4) and death during the first year following PE (HR 3.3; 95\% CI 2.2-5.0) (tables 3 and 4).

TNT remained an independent predictor of mortality after inclusion of the other most important risk factors of death (cancer, immobility and age) in the Cox model: HR 4.0 (95\% CI 2.3-7.1) for in-hospital death and HR 2.8 (95\% CI 1.8-4.3) for 1yr mortality. In the multivariate analysis, cancer at any time was associated with a 3.1 -fold $(95 \%$ CI $2.0-4.8)$ increased risk for 1-yr mortality. Immobility was associated with a HR of 2.4 (95\% CI 1.5-3.8), whereas age was not an independent predictor. Initial haemodynamical instability was defined by

\begin{tabular}{|c|c|c|c|c|c|}
\hline & Parametric rating & Standard error & $\mathrm{p}$-value & Hazard ratio & $95 \% \mathrm{Cl}$ \\
\hline Troponin T & 1.19 & 0.22 & 0.0000 & 3.29 & $2.15-5.02$ \\
\hline Age & 0.02 & 0.01 & 0.0002 & 1.02 & $1.01-1.03$ \\
\hline Cancer & 1.08 & 0.19 & 0.0000 & 2.95 & $2.02-4.29$ \\
\hline \multicolumn{6}{|c|}{ Multivariate analysis } \\
\hline Troponin T & 1.02 & 0.22 & 0.0000 & 2.76 & $1.78-4.28$ \\
\hline Age & 0.00 & 0.01 & 0.7507 & 1.00 & $0.99-1.02$ \\
\hline Cancer & 1.13 & 0.22 & 0.0000 & 3.11 & $2.00-4.82$ \\
\hline Immobility & 0.88 & 0.24 & 0.0002 & 2.41 & $1.52-3.82$ \\
\hline
\end{tabular}


TABLE 4 In-hospital mortality

\begin{tabular}{|c|c|c|c|c|c|}
\hline \multicolumn{6}{|c|}{ Univariate analysis } \\
\hline Age & 0.01 & 0.01 & 0.2968 & 1.01 & $0.99-1.02$ \\
\hline Cancer & 0.59 & 0.26 & 0.0213 & 1.81 & $1.09-2.99$ \\
\hline Immobility & 1.36 & 0.24 & 0.0000 & 3.88 & $2.42-6.22$ \\
\hline Age & -0.01 & 0.01 & 0.1015 & 0.99 & $0.97-1.00$ \\
\hline Cancer & 0.62 & 0.30 & 0.0397 & 1.87 & $1.03-3.39$ \\
\hline Immobility & 1.07 & 0.29 & 0.0002 & 2.90 & $1.65-5.10$ \\
\hline
\end{tabular}

TABLE 5 1-yr mortality after exclusion of patients haemodynamically instable at presentation

\begin{tabular}{|c|c|c|c|c|c|}
\hline \multicolumn{6}{|c|}{ Univariate analysis } \\
\hline Age yrs & 0.03 & 0.01 & 0.0000 & 1.04 & $1.02-1.05$ \\
\hline Cancer & 1.76 & 0.26 & 0.0000 & 5.81 & $3.49-9.69$ \\
\hline Immobility & 1.15 & 0.27 & 0.0000 & 3.15 & $1.85-5.36$ \\
\hline Age yrs & 0.01 & 0.01 & 0.2285 & 1.01 & $0.99-1.03$ \\
\hline Cancer & 1.77 & 0.29 & 0.0000 & 5.90 & $3.32-10.46$ \\
\hline Immobility & 1.11 & 0.31 & 0.0004 & 3.03 & $1.64-5.59$ \\
\hline
\end{tabular}

\section{TABLE 6 1-yr mortality after exclusion of in hospital deaths}

\begin{tabular}{|c|c|c|c|c|c|}
\hline & Parametric rating & Standard error & p-value & Hazard ratio & $95 \% \mathrm{Cl}$ \\
\hline \multicolumn{6}{|c|}{ Univariate analysis } \\
\hline Troponin T & 0.73 & 0.37 & 0.0466 & 2.07 & $1.01-4.23$ \\
\hline Age yrs & 0.05 & 0.01 & 0.0000 & 1.05 & $1.03-1.08$ \\
\hline Cancer & 1.88 & 0.32 & 0.0000 & 6.57 & $3.53-12.23$ \\
\hline Immobility & 0.85 & 0.34 & 0.0137 & 2.33 & $1.19-4.57$ \\
\hline \multicolumn{6}{|c|}{ Multivariate analysis } \\
\hline Troponin $\mathrm{T}$ & 0.49 & 0.37 & 0.1848 & 1.63 & $0.79-3.35$ \\
\hline Age yrs & 0.04 & 0.01 & 0.0093 & 1.04 & $1.01-1.06$ \\
\hline Cancer & 1.92 & 0.37 & 0.0000 & 6.81 & $3.30-14.03$ \\
\hline Immobility & 0.68 & 0.43 & 0.1144 & 1.98 & $0.85-4.61$ \\
\hline
\end{tabular}

resuscitation prior to or at admission, presentation with a systolic blood pressure $<90 \mathrm{mmHg}$ or treatment with catecholamines in the pre-clinical phase. Even after exclusion of initially haemodynamically instable patients TNT was predictive for 1-yr mortality in the multi- and univariate analysis (HR 2.2 (95\% CI 1.2-3.9) and HR 2.8 (95\% CI 1.6-4.9), respectively) (table 5). After the exclusion of patients who died in hospital, TNT only remained predictive in the univariate analysis. After discharge from the hospital, cancer was the strongest predictor for 1-yr mortality (table 6).

Replacement of the data from our prior study [8] by the results for in-hospital outcome from the meta-analysis by BECATTINI et al. [7] led to a shift in the overall odds ratio for death from 


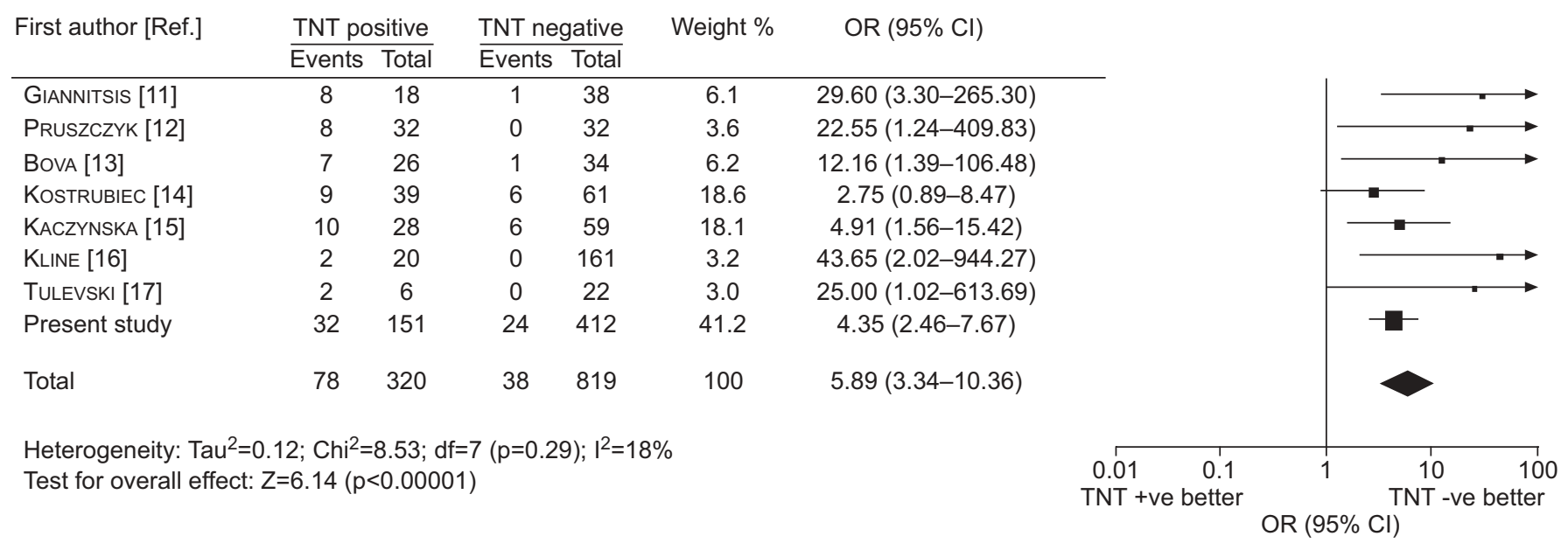

FIGURE 3. Odds ratio for in-hospital mortality based on elevated or normal plasma levels of troponin T (TNT). Levels of TNT after addition of current data to the existing meta-analysis.

7.95 to 5.89 with a reduction in the confidence interval (fig. 3). Due to the current size of the cohort this led to a weight gain from $6.0 \%$ to $41.2 \%$ in the meta-analysis without relevant impact on heterogeneity.

\section{DISCUSSION}

Our study has several interesting findings. First, the fact that elevated TNT is an independent predictor of short-term mortality in PE, as previously suggested by several small studies [8, 11-17], is confirmed in our cohort study. By including the results for in-hospital mortality from the metaanalysis of BECATTINI et al. [7], our study adds a lot of information on the presumed true predictive value of TNT (fig. 3), which might be slightly lower than previously assumed. The odds ratio for in-hospital mortality in the current study is 4.35 (HR 4.32), which is very similar to the odds ratio of 4.01 revealed by pooling 12 studies evaluating troponin I [7]. The best estimate is crucial when it comes to the weighting of risk factors and inclusion in prognostic scores. Such scores are considered to facilitate risk stratification in early patient management, for example, to enable outpatient care for selected low-risk patients [18].

Secondly, to our knowledge, this is the first study evaluating TNT as a predictor for 1-yr mortality in PE. Although TNT seems to be most valuable for mortality prediction in the acute phase, elevated TNT at the time of presentation was also independently predictive for 1-yr outcome (fig. 1a). We included cancer, immobility and age in the multivariate analysis because these factors have previously been reported to be the most important independent risk factors for fatality of PE [9]. LAPORTE et al. [10] prospectively investigated $>15,000$ patients with venous thromboembolism and recently confirmed these three clinical factors as being independently associated with a two to three-fold increased risk for mortality. The pronounced importance of TNT for the early phase was further reflected by a shift from TNT to cancer as a long-term mortality predictor after exclusion of patients who died in hospital (fig. 2b, table 6). However, PE was the most frequent cause of in-hospital and 1-yr mortality in the overall study population, followed by cancer. Clearly, this was not true for
TNT-negative patients, in whom cancer was the primary cause of 1-yr mortality (table 2). Therefore, the reasons for long-term mortality differed in TNT-positive and TNT-negative patients. While nearly all patients dying directly from PE died in hospital, patients dying from cancer were more likely to die after discharge following initial hospitalisation. The median duration of hospitalisation was 11 days and, therefore, longer than described for PE patients in previous studies [19, 20]. The longer hospital stay of TNT-positive patients compared with TNT-negative patients clearly reflects the higher morbidity of TNT-positive patients, who required longer in-patient care.

The epiphenomenon that TNT-positive patients had higher creatinine levels is not surprising since elevated TNT levels have been observed in patients with varying degrees of renal failure [21]. However, cardiac troponins were also shown to be predictive for general mortality in patients with chronic renal failure [22]. In contrast, creatinine levels alone were neither independently predictive for in-hospital nor for 1-yr mortality after inclusion in the Cox model with the covariates TNT, cancer, immobility and age (data not shown).

An interesting observation was that TNT-positive patients tended to experience angina-like chest pain, dyspnoea while resting, tachycardia and syncope, which are suggestive symptoms for right ventricular strain, higher clot burden or central PE. These symptoms were also reported to frequently precede cardiac arrest caused by PE [23]. TNT-negative patients more often experienced pleuritic chest pain (i.e. breathing dependent), dyspnoea on exercise only and cough: symptoms which might reflect pleuritic stimuli by peripheral PE (table 1). The theory of more severe PE in TNT-positive patients is strengthened by objective parameters, such as significantly lower oxygen saturation and higher shock index in these patients.

As patients with elevated troponin levels were more likely to present with severe clinical signs and symptoms of PE, this raises the question of whether TNT adds to the severity assessment, based on clinical presentation data alone. We think that the advantage of TNT is its rapid availability. As an objectively measureable parameter, TNT can be performed in patients who are not able to express themselves or adequately 
describe their symptoms and comorbidities. Most of the clinical PE symptoms are rather unspecific or "soft" parameters, as they are very subjective, interindividually and intraindividually diverse, and might be influenced by social and environmental circumstances. Therefore, it makes sense to add more objective measurable markers to the established haemodynamic parameters. Future prognostic studies might include both hard and soft parameters, but evaluation of the single risk factors will be a premise for weighting. TNT as a hard, objectively measurable parameter with highly prognostic value will surely outweigh several signs and symptoms in such studies.

To date, it is still unclear if positive troponin values in haemodynamically stable patients might identify those who would benefit from thrombolysis. After exclusion of patients who were unstable at presentation we still found a two to three-fold increased risk of 1-yr mortality in TNT-positive patients compared with TNT-negative patients (table 5, fig. 2a). Therefore, although stable patients show a better overall survival, TNT seems to be a useful marker of adverse outcome also in these patients. However, randomised controlled clinical trials are necessary to evaluate the use of lysis in stable patients with elevated TNT.

\section{Conclusion}

Elevated TNT independently predicts in-hospital and 1-yr mortality in patients presenting at an emergency department with acute PE. Elevated troponin levels meant a four-times higher risk of in-hospital death and a three-times higher risk of 1 -yr mortality.

\section{STATEMENT OF INTEREST}

None declared.

\section{REFERENCES}

1 Jeremias A, Gibson CM. Narrative review: alternative causes for elevated cardiac troponin levels when acute coronary syndromes are excluded. Ann Intern Med 2005; 142: 786-791.

2 Kucher N, Rossi E, De Rosa M, et al. Prognostic role of echocardiography among patients with acute pulmonary embolism and a systolic arterial pressure of $90 \mathrm{mmHg}$ or higher. Arch Intern Med 2005; 165: 1777-1781.

3 Torbicki A, Perrier A, Konstantinides S, et al. Guidelines on the diagnosis and management of acute pulmonary embolism: the Task Force for the Diagnosis and Management of Acute Pulmonary Embolism of the European Society of Cardiology (ESC). Eur Heart J 2008; 29: 2276-2315.

4 Becattini C, Vedovati MC, Agnelli G. Diagnosis and prognosis of acute pulmonary embolism: focus on serum troponins. Expert Rev Mol Diagn 2008; 8: 339-349.

5 Jimenez D, Diaz G, Molina J, et al. Troponin I and risk stratification of patients with acute nonmassive pulmonary embolism. Eur Respir J 2008; 31: 847-853.
6 Capstick T, Henry MT. Efficacy of thrombolytic agents in the treatment of pulmonary embolism. Eur Respir J 2005; 26: 864-874.

7 Becattini C, Vedovati MC, Agnelli G. Prognostic value of troponins in acute pulmonary embolism: a meta-analysis. Circulation 2007; 116: 427-433.

8 Janata K, Holzer M, Laggner AN, et al. Cardiac troponin $\mathrm{T}$ in the severity assessment of patients with pulmonary embolism: cohort study. BMJ 2003; 326: 312-313.

9 Nijkeuter M, Sohne M, Tick LW, et al. The natural course of hemodynamically stable pulmonary embolism: clinical outcome and risk factors in a large prospective cohort study. Chest 2007; 131: $517-523$.

10 Laporte S, Mismetti P, Decousus H, et al. Clinical predictors for fatal pulmonary embolism in 15,520 patients with venous thromboembolism: findings from the Registro Informatizado de la Enfermedad TromboEmbolica venosa (RIETE) Registry. Circulation 2008; 117: 1711-1716.

11 Giannitsis E, Muller-Bardorff M, Kurowski V, et al. Independent prognostic value of cardiac troponin $\mathrm{T}$ in patients with confirmed pulmonary embolism. Circulation 2000; 102: 211-217.

12 Pruszczyk P, Bochowicz A, Torbicki A, et al. Cardiac troponin T monitoring identifies high-risk group of normotensive patients with acute pulmonary embolism. Chest 2003; 123: 1947-1952.

13 Bova C, Crocco F, Ricchio R, et al. Importance of troponin $T$ for the risk stratification of normotensive patients with pulmonary embolism. A prospective, cohort study with a three-month follow-up. Haematologica 2005; 90: 423-424.

14 Kostrubiec M, Pruszczyk P, Bochowicz A, et al. Biomarker-based risk assessment model in acute pulmonary embolism. Eur Heart $J$ 2005; 26: 2166-2172.

15 Kaczynska A, Pelsers MM, Bochowicz A, et al. Plasma heart-type fatty acid binding protein is superior to troponin and myoglobin for rapid risk stratification in acute pulmonary embolism. Clin Chim Acta 2006; 371: 117-123.

16 Kline JA, Hernandez-Nino J, Rose GA, et al. Surrogate markers for adverse outcomes in normotensive patients with pulmonary embolism. Crit Care Med 2006; 34: 2773-2780.

17 Tulevski II, ten Wolde M, van Veldhuisen DJ, et al. Combined utility of brain natriuretic peptide and cardiac troponin $\mathrm{T}$ may improve rapid triage and risk stratification in normotensive patients with pulmonary embolism. Int J Cardiol 2007; 116: 161-166.

18 Tapson VF, Huisman MV. Home at last? Early discharge for acute pulmonary embolism. Eur Respir J 2007; 30: 613-615.

19 Kozak LJ, DeFrances CJ, Hall MJ. National hospital discharge survey: 2004 annual summary with detailed diagnosis and procedure data. Vital Health Stat 13, 2006: 1-209.

20 Aujesky D, Stone RA, Kim S, et al. Length of hospital stay and postdischarge mortality in patients with pulmonary embolism: a statewide perspective. Arch Intern Med 2008; 168: 706-712.

21 Hojs R. Cardiac troponin $\mathrm{T}$ in patients with kidney disease. Ther Apher Dial 2005; 9: 205-207.

22 Khan NA, Hemmelgarn BR, Tonelli M, et al. Prognostic value of troponin $\mathrm{T}$ and I among asymptomatic patients with end-stage renal disease: a meta-analysis. Circulation 2005; 112: 3088-3096.

23 Kurkciyan I, Meron G, Sterz F, et al. Pulmonary embolism as a cause of cardiac arrest: presentation and outcome. Arch Intern Med 2000; 160: 1529-1535. 